\title{
Effectiveness of Shortwave Diathermy in Persons with Osteoarthritis of Knee Joint - an Experimental Study
}

\section{Yarusha, Tilak M*, Samuel W, Samuel JD, Babu A, Thomas R and Rebekah G \\ Department of Physical Medicine \&Rehabilitation, Christian Medical College, India}

*Corresponding author: Merlyn Tilak, Department of Physical Medicine \&Rehabilitation, Christian Medical College, Vellore-632 002, India, Tel: 09444463948 ;

Email: merlyntilak@gmail.com

\section{Research Article \\ Volume 2 Issue 1}

Received Date: December 21, 2018

Published Date: February 13, 2019

DOI: $10.23880 /$ aphot-16000120

\section{Abstract}

Background and Objectives: Knee osteoarthritis $(\mathrm{OA})$ is a painful condition affecting 85\% of population with radiologically evident changes in subjects above 65 years of age, causing disability and muscle weakness. Shortwave diathermy (SWD) is a deep heating modality which has been extensively used to alleviate pain in persons with OA knees. However the problem of non-adherence to this modality remains a challenge and a persistent problem because of long treatment period, as a result of which, substantial numbers of patients do not get the maximum benefit, leading to poor health outcomes. Therefore the purpose of this study was to find out if shorter periods of shortwave diathermy in persons with knee osteoarthritis improve outcomes and compliance to therapy.

Design: Quasi experimental pre-test post- test study.

Methodology: A total of 15 subjects with OA knee, mean age of $57.4 \pm 5$ years consented to participate in this study after a written consent. The baseline assessment was performed using Visual Analogue Scale (VAS), Western Ontario and McMaster Universities Osteoarthritis Index (WOMAC) and Elderly Mobility Scale (EMS) for assessing pain, activity and mobility status respectively. After 5 days of SWD and Quadriceps strengthening exercises, a final assessment was done to document the change in VAS, WOMAC and EMS. This data was analyzed to find out if outcomes improved with short period of SWD. The number of drop outs was also analyzed to report the patient compliance to therapy.

Results: The results from this study shows significant reduction in pain using VAS (p value $=0.003$ ), improvement in WOMAC scores ( $p$ value $=0.002$ ) and EMS ( $p$ value $=0.033$ ), following 5 days of SWD. The dropout rate due to noncompliance was $6.5 \%$.

Conclusion: There was significant reduction in pain, and improvement in activity level and mobility status following short period of SWD in subjects with OA knees with a low drop out percentage.

Keywords: Knee; Degenerative Joint Disease; Shortwave Diathermy 


\section{Annals of Physiotherapy \& Occupational Therapy}

Abbreviations: OA: Osteoarthritis; SWD: Shortwave diathermy; VAS: Visual Analogue Scale; WOMAC: Western Ontario and McMaster Universities Osteoarthritis Index; EMS: Elderly Mobility Scale.

\section{Introduction}

Osteoarthritis $(\mathrm{OA})$ of knee is a chronic degenerative joint disorder and is considered as one of the most common musculoskeletal disorders leading to pain and discomfort [1,2]. Almost $85 \%$ of subjects nearing 60 years of age present with radiographic evidence of osteoarthritis $[3,4]$. It has been considered as a "wear and tear" disease leading to loss of cartilage [5]. However, this painful and disabling joint disease is not localized to cartilage alone but is considered as a chronic disease of the whole joint, including articular cartilage, meniscus, ligament, and peri-articular muscle that may result from multiple patho-physiological mechanisms [6].

A wide range of treatments for $\mathrm{OA}$ Knee have been discussed in the literature [5-11]. Among these shortwave diathermy (SWD), a deep heating modality has been reported to have a measurable effect on pain reduction in persons with OA knee [10-29].

Application of SWD to the involved tissues has been reported to increase vascular circulation and change tissue temperature, which directly results in vascular dilatation, an increase in pain threshold, and a decrease in pain and swelling [10-26]. The vascular improvement accelerates the inflammatory process by increasing nutrition and oxygen supply and by removing metabolic waste products. In addition, SWD have also been shown to reduce joint stiffness, stimulate connective tissue repair, and reduce muscle spasm and pain [27-29].

Despite the many beneficial effects reported in literature, the problem of non-adherence to this modality remains a challenge and a persistent problem because of long treatment period, as a result of which, substantial numbers of patients do not get the maximum benefit, leading to poor health outcomes [22,30-33].

Jan $\mathrm{MH}$, et al. [22] in their study had given SWD for 8 consecutive weeks with a $40 \%$ drop out rate. [22] Similar study done by Fukuda TY, et al. [30] where the subjects with OA Knees received SWD for 3 consecutive weeks with a $43 \%$ drop out rate [30]. Both the studies along with many more in the literature have shown a large percentage of drop outs using extensive treatment periods of SWD for OA knees [22,30-33].

Tilak M, et al. Effectiveness of Shortwave Diathermy in Persons with Osteoarthritis of Knee Joint - an Experimental Study. Ann Physiother Occup Ther 2019, 2(2): 000120.
Although several studies report that SWD can be used to reduce pain and swelling, accelerate the inflammation process and promote healing in tissues with chronic inflammation [13,15-22] till date there are no reports on the effectiveness of SWD when given for shorter periods. Hence in this study we aim to find out if shorter periods of shortwave diathermy in persons with knee osteoarthritis improve outcomes and the compliance to therapy.

\section{Method}

\section{Design Overview}

This Quasi experimental pre-test post- test study was done on persons with $\mathrm{OA}$ knees who were selected by convenient sampling method, in which the outcome assessor was blinded to the baseline assessment of the subjects.

\section{Setting and Participants}

The study was carried out in the Physiotherapy outpatient unit of Christian Medical College, Vellore, Tamilnadu, India. Persons with OA knee were assessed for eligibility, and a written consent was obtained by the principal investigator. Persons diagnosed with OA knee by the Orthopaedician at Christian Medical College, Vellore, between the age 50 and 70 years of both genders were considered to be eligible for inclusion. Persons with open wound or haemorrhage at the affected knee, metallic implants at knee joint, disturbed skin sensation in the involved leg, venous thrombosis and arterial diseases involving the lower limbs, tumours in the involved limb and epileptic patients were excluded from the study. Approval for the study was obtained from the Institutional Review board (Research and ethics committee) of the Christian Medical College, Vellore.

\section{Intervention}

The subjects were made to sit in a comfortable chair with a back and arm rest. The limb with OA knee was kept in 90-90 position with foot resting on a low stool. The subjects were instructed not to move, not to sleep and not to touch any cables. The electrodes were positioned over the medial and lateral aspect of the knee (contra planar method) and medium spacing was used. The subjects were asked to inform if they felt anything more than the warmth felt when they blew on the dorsum of their hand. Intensity was adjusted according to the warmth felt by the patients at the knee. Treatment was given for 20 minutes following which knee strengthening exercises were taught. They were also given a pictorial exercise hand out and advised to follow the exercises two times in a day. 


\section{Annals of Physiotherapy \& Occupational Therapy}

\section{Measurement Instruments and Outcome Measures}

The participant's data sheet included demographic data and outcome scores. A baseline assessment was done including pain score, activity level and mobility status using VAS, EMS and WOMAC respectively. After this the patient was seated in a comfortable chair and SWD was given for 20 minutes following which knee strengthening exercises were taught. They were also given a pictorial exercise hand out and advised to follow the exercises two times in a day. After 5 days a final assessment was done using VAS, WOMAC and EMS for comparison. The data collected was analyzed to find out if outcomes improved with SWD. The description of each scale is given below.

Visual Analogue Scale (VAS): The VAS is a reliable, valid, responsive, and frequently used pain outcome measure $[34,35]$. It consists of a bidirectional $10 \mathrm{~cm}$ straight line with two labels, that is, "no pain" and "worst possible pain", located at either end of the line. Patients were instructed to draw a vertical mark on the line indicating their pain level [34].

The Western Ontario and McMaster Universities Arthritis Index (WOMAC): WOMAC is widely used in the evaluation of Hip and Knee Osteoarthritis. It is a selfadministered questionnaire consisting of 24 items divided into 3 subscales; pain, stiffness and physical function [36]. The test questions are scored on a scale of 0-4, which correspond to: None, Mild, Moderate, Severe, and Extreme. Higher scores on the WOMAC indicate worse pain, stiffness, and functional limitations $[36,37]$.
Elderly Mobility Scale (EMS): The EMS is a 20 point validated assessment tool for the assessment of frail elderly subjects [38]. EMS evaluates an individual's mobility problems through seven functional activities including bed mobility, transfers and bodily reaction to perturbation [39]. In EMS gait is assessed based on the type of assistance required to walk. Subjects who score above 14 are generally able to perform mobility manoeuvres alone and safely and are independent in basic ADL.

\section{Statistical Analysis}

The pain, activity level and mobility status were measured using VAS, EMS and WOMAC respectively. Statistical analysis was done using Wilcoxon Signed Rank Test to compare the significance between the pre and post assessment scores. Data analysis was done using SPSS 16.0.

\section{Results}

A total of 15 subjects with $\mathrm{OA}$ knee consented to participate in this study. The mean age of the participants was $57.4 \pm 5$ years with a majority of females $(73.33 \%)$. The mean height and weight of the participants were $161.53 \pm 10.42 \mathrm{cms}$ and $65.4 \pm 9.57 \mathrm{Kgs}$ respectively. The demographic characteristics of the participants and the baseline VAS score, WOMAC and EMS are shown in Table 1. There were 2 dropouts out of 15 subjects who consented for the treatment. One was sick and the other not compliant to therapy. Hence the total drop out percentage was $13 \%$ and the drop out due to noncompliance was $6.5 \%$.

\begin{tabular}{|c|c|c|}
\hline \multicolumn{2}{|c|}{ Participants characteristics } & Initial assessment \\
\hline Age & $\mathrm{N}=15$ & $57.4 \pm 5$ \\
\hline Weight & $\mathrm{N}=15$ & $65.4(9.57)$ \\
\hline Height & $\mathrm{N}=15$ & $161.53(10.42)$ \\
\hline \multirow{2}{*}{ Gender } & Female & $11(73.33)$ \\
\cline { 2 - 3 } & Male & $4(26.67)$ \\
\hline Pain & Baseline scores: \\
\hline Activity level & VAS & $6.4 \pm 1.7$ \\
\hline Mobility status & WOMAC & $66.02 \pm 17.99$ \\
\hline
\end{tabular}

VAS, Visual Analogue Scale; WOMAC, The Western Ontario and McMaster Universities Arthritis Index. EMS, Elderly Mobility Scale.

Categorical variables are expressed as number (\%), continuous variables are expressed as mean \pm SD

Table 1: Demographic characteristics and outcomes of participants at baseline ( $\mathrm{N}=15)$.

The mean pain intensity of the participants was $6.4 \pm$ 1.7 out of 10 in the VAS before starting the therapy. SWD was given as shown in Figure 1, after the baseline data was collected. The effect of SWD was measured using VAS, 


\section{Annals of Physiotherapy \& Occupational Therapy}

WOMAC and EMS, after 5 days of SWD and Quadriceps strengthening exercises and the mean scores are shown in Table 2.

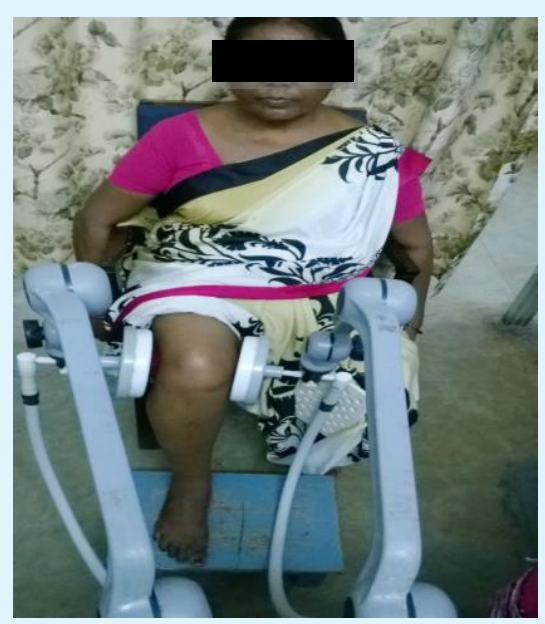

Figure 1: Person with OA knee receiving Shortwave Diathermy.

\begin{tabular}{|c|c|c|c|}
\hline Outcomes & $\begin{array}{c}\text { Pre-treatment } \\
\text { (Baseline, } \\
\text { N=15) }\end{array}$ & $\begin{array}{c}\text { Post-treatment } \\
\text { (Final, N=13) }\end{array}$ & Significance \\
\hline Pain (VAS) & $6.4 \pm 1.7$ & $4.38 \pm 1.98$ & $0.003^{*}$ \\
\hline $\begin{array}{c}\text { Activity } \\
\text { level } \\
\text { (WOMAC) }\end{array}$ & $66.02 \pm 17.99$ & $46.71 \pm 23.65$ & $0.002^{*}$ \\
\hline $\begin{array}{c}\text { Mobility } \\
\text { (EMS) }\end{array}$ & $18.85 \pm .99$ & $19.46 \pm 1.2$ & 0.033 \\
\hline
\end{tabular}

*Statistically significant (P value $<0.01$ )

Table 2: Pre- post comparison of pain (VAS), Activity level (WOMAC) and Mobility status (EMS) using Wilcoxon Signed Rank Test.

The baseline score for VAS and WOMAC was $6.4 \pm 1.7$ and $66.02 \pm 17.99$ which reduced significantly to $4.38 \pm 1.98$ and $46.71 \pm 23.65$ respectively after 5 days of SWD. (P value $=0.003,0.002$ respectively) However, there was no significant improvement in the EMS score. $(\mathrm{P}$ value = 0.033)

\section{Discussion}

The objective of this study was to find out the short term effects of short wave diathermy in patients with osteoarthritis of knee and their compliance to therapy. A total of 15 subjects with OA knee consented to participate in this study.

Tilak M, et al. Effectiveness of Shortwave Diathermy in Persons with Osteoarthritis of Knee Joint - an Experimental Study. Ann Physiother Occup Ther 2019, 2(2): 000120.
The descriptive statistics was done to find the baseline characteristics of the subjects who participated in this study. Pain was assessed using VAS, activity level using WOMAC and mobility status using EMS. Their baseline VAS was $6.4 \pm 1.7$, WOMAC was $66.02 \pm 17.99$ and EMS was $18.85 \pm .99$ (Table 1 ).

The subjects received 20 minutes of SWD for 5 consecutive days. Out of the 15 subjects, 13 completed the study while 2 subjects dropped out on the fourth and fifth day due to non-compliance and sickness respectively.

On the 5th day a final assessment was done for pain, mobility status and activity using VAS, EMS and WOMAC respectively. There was considerable reduction in pain after 5 days of treatment (4.38 \pm 1.98$)$; the mean value of WOMAC was $46.71 \pm 23.65$ and the mean EMS score was 19.46 \pm 1.2 (Table 2).

The data was analyzed for significance using Wilcoxon Signed Rank Test. The results show that there is a significant reduction in pain after 5 days of SWD. (P value $=0.003)$ This correlates and supports the previous studies which have found significant reduction in pain after SWD application (Table 2) [22-29]. Funda CA, et al (2012) found shortwave diathermy along with exercise to have best outcomes in the treatment of knee OA [40]. SWD produces an oscillating electromagnetic field, which causes movement of ions, distortion of molecules, and creation of eddy currents, which in turn results in heat production in the deep tissue [14]. This may be the potential mechanism which leads to improvement in outcomes, as an increase in the tissue temperature causes vascular dilatation, increase in pain threshold, and decrease in pain and swelling [10,13,18,23-26]. The vascular improvement in turn may accelerate the inflammatory process by increasing nutrition and oxygen supply and by removing metabolic waste products. In addition, deep heating may also reduce joint stiffness, stimulate connective tissue repair, and reduce muscle spasm, thereby improving outcomes after application of SWD [27-29].

However the problem of non-adherence to this modality remained a challenge and a persistent problem because of long treatment period, as a result of which, substantial numbers of patients did not get the maximum benefit, leading to poor health outcomes [22-30].

Jan $\mathrm{MH}$, et al. [22] in their study had reported significant reduction in pain for patients who received 5 sessions of SWD per week for 8 consecutive weeks [22]. 


\section{Annals of Physiotherapy \& Occupational Therapy}

Similar study done by Fukuda TY, et al. [30] where subjects with OA Knees received 3 sessions per week of SWD for 3 consecutive weeks also showed a significant reduction in pain [30]. However, the treatment protocol was long and exhaustive, which may have led to patient non-compliance with a high dropout rate of almost $40 \%$ in both these studies $[22,30]$.

In an attempt to minimize the drop outs arising out of non-compliance, we designed this study using a shorter protocol. The five days of SWD protocol still yielded an excellent result with a significant reduction in pain, and improvement in their activity level following the reduction in pain. ( $\mathrm{P}$ value $<0.01$ ) The mobility status however did not show a significant improvement as the subjects were already ambulant. $(\mathrm{P}$ value $=0.03$ ) The dropout rate was also considerably low (13\%), using this protocol, and the drop out due to non-compliance was only $6.5 \%$.

There were some limitations in this study; first, the time when the symptoms improved was not documented; second, non treatment or placebo group (control) was not included as it would be unethical to deny treatment for the subjects who come for SWD; third, this protocol of treatment for 5 days was not compared against any wellknown protocol to find out if they were comparable as there were no short term protocols available in literature.

This study is the first of its kind to use a shorter period of SWD for treating subjects with OA knees. The results have shown that pain reduces and the activity level increases significantly using shorter periods of SWD for OA knees. The results from this study can be used by clinicians and therapists while planning and implementing treatment protocol for OA knees, with good patient compliance. However, it is not known from this study if the effectiveness lasts for a longer duration. Therefore substantiation with long term follow up is necessary to find its long term effectiveness as $\mathrm{OA}$ is a progressive degenerative disease. Future studies can focus on minimizing bias by randomizing and blinding at allocation and assessment. In addition, control groups using other modalities and combinations of therapies can be explored.

\section{Conclusion}

The results from this study suggest that shorter periods of SWD in persons with knee OA may improve outcomes and patient compliance to therapy.
Acknowledgements: We would like to thank the Christian Medical College, Vellore for the Institutional Fluid Research Grant.

\section{References}

1. Bennell KL, Egerton T, Wrigley TV, Hodges PW, Hunt $M$, et.al. (2011) Comparison of neuromuscular and quadriceps strengthening exercise in the treatment of varus malaligned knees with medial knee osteoarthritis: a randomized controlled trial protocol. BMC Muscloskeletal Disorders 12: 276.

2. Hunter DJ, Sharma L, Skaife T (2009) Alignment and osteoarthritis of knee. J Bone Joint Surg Am 1(1): 8589.

3. Cooper C (1994) Inc: Rheumatology. In: Klippel J \& Dieppe $P$ (Eds.), CV Mosby The epidemiology of osteoarthritis. New York, pp: 1-4.

4. Osiri M, Welch V, Brosseau L, Shea B, McGowan J, et al. (2000) Transcutaneous electrical nerve stimulation for knee osteoarthritis. Cochrane Database Syst Rev 4: CD002823.

5. Berenbaum F (2013) Osteoarthritis as an inflammatory disease (osteoarthritis is not osteoarthrosis!). Osteoarthritis Cartilage 21(1): 1621.

6. Hayami T (2008) Osteoarthritis of the knee joint as a cause of musculoskeletal ambulation disability symptom complex (MADS). Clin Calcium 18(11): 1574-1580.

7. (2000) Recommendations for the medical management of osteoarthritis of the hip and knee: 2000 update. American College of Rheumatology Subcommittee on Osteoarthritis Guidelines. Arthritis Rheum 43(9): 1905-1915.

8. Zhang W, Nuki G, Moskowitz RW (2010) OARSI recommendations for the management of hip and knee osteoarthritis: part III: Changes in evidence following systematic cumulative update of research published through January 2009. Osteoarthritis Cartilage 18(4): 476-499.

9. Reginster JY, Pelletier JP, Martel-Pelletier J, Henrotin $Y$ (1999) Osteoarthritis clinical and experimental aspects. Berlin: Springer-Verlag, pp: 453-479. 
10. Jan MH, Lai JS (1991) The effects of physiotherapy on osteoarthritic knees of females. J Formos Med Assoc; 90(10): 1008-1013.

11. Kloth LC, Ziskin MC (1990) Diathermy and pulsed electromagnetic fields. Inc: Michlovitz (Ed.s), PA: FA Davis Company, Philadelphia, pp: 170-199.

12. Svarcova J, Trnavsky K, Zvarova J (1987) The influence of ultrasound, galvanic currents and shortwave diathermy on pain intensity in patients with osteoarthritis. Scand J Rheumatol Suppl. 67: 8385.

13. Jan MH, Yip PK, Lin KH (1993) Change of arterial blood flow and skin in temperature after direct and indirect shortwave heating on knee. Formosan Journal of Physical Therapy 18: 64-71.

14. Goats GC (1989) Pulsed electromagnetic (short-wave) energy therapy. Br J Sports Med 23(4): 213-216.

15. Haralson K (1988) Physical modalities. In: Banwell BF, Gall V (Eds.), Physical Therapy Management of Arthritis Clinics in Physical Therapy Series. Churchill Livingstone New York, pp: 77-106.

16. Balogun JA, Okonofua FE (1988) Management of chronic pelvic inflammatory disease with shortwave diathermy. Phys Ther 68(10): 1541-1545.

17. Goats GC (1989) Continuous short-wave (radiofrequency) diathermy. Br J Sports Med 23(2): 123127.

18. Rennie GA, Michlovitz SL (1996) Biophysical principles of heating and superficial heating agents. In: Michlovitz SL (Ed.), Thermal Agents in Rehabilitation. $3^{\text {rd }}$ (Edn.), PA: FA Davis Company, Philadelphia, pp: 107-138.

19. Lehmann JF, DeLateur BJ (1982) Therapeutic heat. In: Lehmann JF (Eds.), 'Therapeutic Heat and Cold'. $3^{\text {rd }}$ (Edn.), Williams and Wilkins, Baltimore.

20. Kloth, L (1986) Shortwave and microwave diathermy. In: 'Thermal Agents in Rehabilitation' Michlovitz, SL \& Wolf SL (Eds.), FA Davis Company, Philadelphia, pp: 177-216.

21. Wright V (1964) Treatment of osteoarthritis of the knees. Ann Rheum Dis 23(5): 389-391.

Tilak M, et al. Effectiveness of Shortwave Diathermy in Persons with Osteoarthritis of Knee Joint - an Experimental Study. Ann Physiother Occup Ther 2019, 2(2): 000120.
22. Jan MH, Chai HM, Wang CL, Lin YF, Tsai LY, et al. (2006) Effects of repetitive short wave diathermy for reducing synovitis in patients with knee osteoarthritis: an ultrasonographic study. Physical therapy 86(2): 236-244.

23. Hecox B (1994) Physiological response to local heat gain or loss. In: Hecox B, Mehreteab TA, Weisberg J (Eds.), Physical Agents: A Comprehensive Text for Physical Therapists. Conn: Appleton, Lange East Norwalk, pp: 91-114.

24. Kitchen S, Partridge C (1992) Review of shortwave diathermy continuous and pulsed patterns. Physiotherapy 78(4): 243-252.

25. Kloth LC, Ziskin MC (1990) Diathermy and pulsed electromagnetic fields. In: Michlovitz SL (Eds.), Thermal Agents in Rehabilitation. PA: FA Davis Company, Philadelphia, pp: 170-199.

26. Svarcova J, Trnavsky K, Zvarova J (1987) The influence of ultrasound, galvanic currents and shortwave diathermy on pain intensity in patients with osteoarthritis. Scand J Rheumatol Suppl 67: 8385.

27. Lehmann JF, Masock AJ, Warren CG, Koblanski JN (1970) Effect of therapeutic temperatures on tendon extensibility. Arch Phys Med Rehabil 51(8): 481-487.

28. Lehmann JF, McMillan JA, Brunner GD, Blumberg JB (1959) Comparative study of the efficiency of shortwave, microwave and ultrasonic diathermy in heating the hip joint. Arch Phys Med Rehabil 40: 510512.

29. Svarcova J, Trnavsky K, Zvarova J (1988) The influence of ultrasound, galvanic currents and shortwave diathermy on pain intensity in patients with osteoarthritis. Scand J Rheumatol 17 (sup67): 83-85.

30. Fukuda TY, Alves da Cunha R, Fukuda VO, Rienzo FA, Cazarini C, et al. (2011) Pulsed shortwave treatment in women with knee osteoarthritis: a multicenter, randomized, placebo-controlled clinical trial. Physical Therapy 91(7): 1009-1017.

31. Cetin N, Aytar A, Atalay A, Akinan MN (2008) Comparing hot pack, shortwave diathermy, ultrasound and TENS on isokinetic strength, pain and functional status of women with osteoarthritis knee: 


\section{Annals of Physiotherapy \& Occupational Therapy}

a single blind, randomized, controlled trial. Am J Phys Med Rehabil 87(6): 443-451.

32. Laufer Y, Zilberman R, Porat R, Nahir AM (2005) Effects of pulsed shortwave diathermy on pain and functions of subjects with osteoarthritis of knee: a placebo- controlled double blind clinical trial. Clin Rehabil 19(3): 255-263.

33. Rattanachaiyanout M, Kuptniratsaikul V (2008) No additional benefits of shortwave diathermy over exercise program for knee osteoarthritis in peri or post menopausal women:an equivalence trial. Osteoarthritis Cartilage 16(7): 823-828.

34. Hjermstad MJ, Fayers PM, Haugen DF (2011) Studies comparing numerical rating scales, verbal rating scales, and visual analogue scales for assessment of pain intensity in adults: a systematic literature review. J Pain Symptom Manage. 41(6): 1073-1093.

35. Ferraz MB, Quaresma MR, Aquino LR, Atra E, Tugwell P, et al. (1990) Reliability of pain scales in the assessment of literate and illiterate patients with rheumatoid arthritis. J Rheumatol 17(8): 1022-1024.
36. American College of Rheumatology. Western Ontario and McMaster Universities Osteoarthritis index (WOMAC).

37. Roos EM, Klassbo M, Lohmander LS (1999) WOMAC osteoarthiritis index: Reliability, Validity and responsiveness in patients with arthroscopically assessed osteoarthritits. Scand J Rheumatol 28(4): 210-215.

38. Smith R (1994) Validation and Reliability of the Elderly Mobility Scale. Physiotherapy 80 (11): 744747.

39. Chiu AY, Au-Yeung SS, Lo SK (2003) A comparison of four functional tests in discriminating fallers from non-fallers in older people. Disabil Rehabil 25(1): 4550 .

40. Atamaz FC, Durmaz B, Baydar M, Demircioglu OY, Iyiyapici A, et al. (2012) Comparison of the Efficacy of Transcutaneous Electrical Nerve Stimulation, Interferential Currents, and Shortwave Diathermy in Knee Osteoarthritis: A Double-Blind, Randomized, Controlled, Multicenter Study. Arch Phys Med Rehabil 93(5): 748-756. 